\section{CORRECTION}

DOI: $10.1039 / \mathrm{cta}$ ta90041g www.rsc.org/MaterialsA The correct author list is as follows;

\title{
Correction: Magnetic yolk-shell mesoporous silica microspheres with supported Au nanoparticles as recyclable high-performance nanocatalysts
}

\author{
Qin Yue, Yu Zhang, Can Wang, Xiqing Wang, Zhenkun Sun, Xiu-Feng Hou,
} Dongyuan Zhao and Yonghui Deng*

Correction for 'Magnetic yolk-shell mesoporous silica microspheres with supported Au nanoparticles as recyclable high-performance nanocatalysts' by Yonghui Deng et al., J. Mater. Chem. A, 2015, DOI: 10.1039/c4ta06967f.

There is an error in the author list provided in the original manuscript. The third author should be 'Can Wang' not 'Chun Wang'.

Qin Yue, Yu Zhang, Can Wang, Xiqing Wang, Zhenkun Sun, Xiu-Feng Hou, Dongyuan Zhao and Yonghui Deng* The Royal Society of Chemistry apologises for these errors and any consequent inconvenience to authors and readers. 